\title{
Editorial
}

\section{Reformando la educación médica para una atención integral y con calidad}

\section{Reforming medical education for an integral care with quality}

\author{
Manuel L. Núñez-Vergara ${ }^{1}$, Carlos Saavedra-Leveau ${ }^{1}$ \\ ${ }^{1}$ Facultad de Medicina, Universidad Nacional Mayor de San Marcos, Lima, Perú
}

Correspondencia:

Manuel L. Nuñez-Vergara

mnunezv@unmsm.edu.pe

Recibido: 23 agosto 2017

Aceptado: 29 agosto 2017

Conflictos de interés: Ninguno con el presente editorial.

Citar como: Manuel L. Núñez-Vergara, Carlos Saavedra-Leveau. Editorial. Reformando la educación médica para una atención integral y con calidad. An Fac med. 2017;78(3): 261-262

DOI: $h t t p: / / d x . d o i . o r g / 10.15381 /$ anales.v78i3.13755
Son innumerables los avances en el desarrollo social y la práctica médica, pudiéndose constatar que muchos de los flagelos de ayer, particularmente aquellos asociados a las condiciones de vida y enfermedades infecciosas, han retrocedido significativamente. Sin embargo, importantes segmentos de nuestra población aun no encuentran una atención integral a sus problemas de salud, lo que además se ve asociado, por ejemplo, a la presencia de nuevos agentes infecciosos, reconfiguraciones demográficas y epidemiológicas, transformaciones sociales y riesgos ambientales que emergen o recrudecen, cambios que no han sido acompañados necesariamente al mismo paso por avances en la educación de los profesionales de la salud en nuestras facultades ${ }^{(1)}$. En varias ocasiones, más allá de discursos y esfuerzos, hemos de reconocer que nuestras aulas han sido testigos de reformas, sin cambios ${ }^{(2)}$ en la esencia o inclusive en la forma con que nuestros estudiantes aprenden.

Han transcurrido cerca de 500 años de enseñanza médica en el Perú, iniciada con la creación de las cátedras clásicas de medicina en la Universidad de San Marcos, las cuales enfrentaron procesos de reforma y modernización con Hipólito Unanue a fines del siglo XVIII y Cayetano Heredia en la segunda mitad del siglo XIX (Salaverry, 2006; Salaverry, 2014). Hoy, y con giros menores en el camino, San Fernando se reencuentra nuevamente con el reto de cambiar, recogiendo el trabajo y legado que la han convertido en una institución líder en la enseñanza médica en el Perú, pero proyectándose hacia el siglo XXI con una propuesta formativa transformadora ${ }^{(1)}$.
Esta reforma se da en un contexto particular, en la medida en que, por un lado, se ve catalizada por una Ley Universitaria, que incorpora Estudios Generales como una primera etapa previa de la formación universitaria; pero al mismo tiempo, se ve enriquecida en San Fernando en particular, al incluir a 5 escuelas de ciencias de la salud (medicina, enfermería, tecnología médica, nutrición y obstetricia), generando un escenario de desafíos, pero sobre todo de oportunidades.

San Fernando enfrenta así el reto de desarrollar una reforma de tercera generación, sobre las bases de reformas de primera (currículo basada en la ciencia) y segunda (currículo basado en problemas) generación que no siempre lograron consolidarse. Esta reforma ha de promover la formación de médicos capaces de desenvolverse en escenarios diversos y dinámicos, que recojan con capacidad de análisis crítico lo mejor del conocimiento global y se desenvuelvan de manera ética en una profesión que enfrenta crecientes retos.

Como ayer, la reforma requiere y pretende cuestionar el por qué y la forma en que enseñamos en salud. Ante esto, son tres los pilares que deben sustentarla: en primer lugar, un empate muy firme con el perfil requerido en los escenarios de desempeño laboral, particularmente aquellos en nuestro país, que demandan que se coloque a la persona en primer lugar (5); en segundo lugar, es crítico que todos los diseños, recursos y acciones estén alineados y articulados tras el perfil establecido, generando sinergias institucionales, eficiencia en el empleo de los recursos y eficacia en el logro de los objetivos, incluyendo por lo tanto el romper paradigmas, caminando 
a un rol del docente como facilitador en la exploración del conocimiento y al alumno como protagonista en busca del conocimiento. En tercer lugar, debe recoger en su diseño, los elementos sustanciales necesarios para una formación integral, centrada en el estudiante, que articule las ciencias básicas y clínicas, que permita una inserción temprana en los espacios de práctica profesional; que estructure una formación interdisciplinaria, rompiendo los estanques existentes; que se enfoque en una formación y evaluación por competencias, particularmente aquellas vinculadas a la atención primaria; con un empleo intensivo, pero con pertinencia, de los nuevos recursos de la información y la comunicación; pero sobre todo, que reconozca en la conducta ética y el respeto a la diversidad cultural, condiciones innatas de la práctica médica.

Por lo tanto, el desafío no es tan solo formar profesionales, sino ciudadanos que se sientan comprometidos con los problemas de nuestro sistema de salud, y sobre todo parte protagónica de las soluciones que requerimos construir como país, reconociendo en la salud un derecho humano fundamental ${ }^{(6)}$. Esto implica que partamos desde la misma vocación con que llega el estudiante, aquel "amor por cuidar de modo integral la salud del prójimo, más aun en situación de enfermedad o sufrimiento ... un proceso humano que nacerá, crecerá y continuará su existencia, siempre y cuando goce de un ambiente o entorno que la nutra y estimule" (7). Se trata, en última instancia, de generar las condiciones propicias para una formación en que se desarrollen y asienten valores y principios, una forma de ver la vida y la práctica médica, y ubicarse como agente de desarrollo social.

Sin embargo, el desafío es mayor, porque una reforma curricular del nivel y alcance que se pretende realizar, requiere necesariamente una reforma institucional, un rediseño de la infraestructura y renovación del equipamiento, el fortalecimiento de los cuadros docentes, pero, sobre todo, y posiblemente lo que más esfuerzo exigirá, el desarrollo de una cultura que sustente esta reforma. Así, se trata de construir una visión compartida de un proyecto institucional que sea asumido por la comunidad sanfernandina ${ }^{(8)}$, como un referente estratégico sobre el cual avanzar, y que tenga la capacidad de trascender personas y gestiones.
Más aun, San Fernando es patrimonio y estandarte de la nación, y de la educación médica en particular; el éxito de su reforma ha de tener la capacidad de motivar y cimentar cambios en otras instituciones universitarias. Esta responsabilidad, hoy reafirmada al asumir el decano de nuestra Facultad la conducción de la Asociación Peruana de Facultades de Medicina (ASPEFAM), implica sumar esfuerzos, compartir experiencias y aprender mutuamente con las 24 facultades de medicina con que convergemos en ASPEFAM y, más aun, con las 46 que actualmente funcionan en el país.

Son retos que requieren ser asumidos con todos los miembros de esta Casa de Estudios, incluyendo a aquellos egresados, que se han formado en nuestras aulas, en el pre y posgrado, porque no estamos tan solo embarcados en llevar adelante una reforma curricular, sino más importante aún, en el esfuerzo por contribuir desde la educación médica a hacer realidad el derecho que tenemos todos los peruanos a una atención integral y con calidad.

\section{REFERENCIAS BIBLIOGRÁFICAS}

1. Frenk J, Chen L, Bhutta ZA, Cohen J, Crisp N, Evans T, et al. Health professionals for a new century: transforming education to strengthen health systems in an interdependent world. Lancet. 2010 Dec 4;376:1923-58. doi:10.1016/S0140- 6736(10)61854-5.

2. Bloom SW. Structure and ideology in medical education - an analysis of resistance to change. J Heal Soc Behav. 1988;29(4):294-306.

3. Salaverry O. Una visión histórica de la educación médica. An Fac Med 1998 Abr 7; 59(3):215-9. doi:http://dx.doi.org/10.15381/anales.v59i3.4623.

4. Salaverry O. El inicio de la educación médica moderna en el Perú . La creación de la Facultad de Medicina de San Fernando. Acta Médica Peru. 2006;23(2):122-31.

5. Sen A. Primero la gente: una mirada desde la ética del desarrollo a los principales problemas del mundo globalizado. Bilbao: Ediciones Deusto; 2007.

6. Miranda JJ, Yamin AE. Educando a los futuros profesionales de la salud: un reto para la salud y los derechos humanos en América Latina. Rev Peru Med Exp Salud Publica. 2008;25(3):302-8.

7. Perales A, Mendoza A, Sánchez E. Vocación médica en médicos de pres tigiada conducta profesional. An Fac Med. 2013 May 21;74(4):291-300. doi:http://dx.doi.org/10.15381/anales.v74i4.2700.

8. Murillo JP, Franco G. San Fernando ¿Utopia o proyecto inconcluso? An Fac Med. 2008 Jul 10;69(2):130-9. DOI: http://dx.doi.org/10.15381/anales. v69i2.1157. 